\title{
Spatiotemporal Modeling in Economics
}

\section{Orlando Gomes*}

Lisbon Higher Institute of Accounting and Administration (ISCAL/IPL), Av. Miguel Bombarda 20, 1069-035 Lisbon, Portugal

Economics, as any other science, resorts to the temporal and to the spatial dimensions in order to build a structure for the problems it typically addresses. In particular, time is of crucial importance, given the peculiar nature of economic problems; agents, located at any initial point in time, $t=0$, set plans for the future, taking into account a more or less distant horizon. It is in this kind of setting that households maximize utility and firms maximize profits, choosing optimal trajectories for consumption, for the allocation of the labor input, for prices or for traded quantities of goods and services.

Furthermore, dealing with time implies attributing a central role to uncertainty; future unknown events force economic agents to generate forecasts and it is precisely the forward-looking character of Economics and the consequent relevance of expectations that constitutes a major distinctive feature of this field of knowledge.

Naturally, the spatial dimension has also a prominent role in Economics. International trade, capital flows or the diffusion of knowledge occurs in the physical space and, therefore, this must be a fundamental piece of an integrated economic analysis. The effects exerted by centripetal and centrifugal forces shape how markets evolve, how beliefs disseminate and how the economic system is constantly reshaped.

An effort to merge both dimensions seems, thus, a logical step in developing a more robust economic science, and steps in this direction have been undertaken in the last few years. A good example of a contemporaneous economic theory built along the two mentioned dimensions is the framework proposed by Lucas [1] and Comin et al. [2] concerning the diffusion of ideas. In these authors' setup, ideas or knowledge diffuse through social interactions with agents meeting randomly with each other; when one agent possessing knowledge meets other agent that has not yet accessed the idea, there is a process of knowledge transmission. This is a purely mechanical process of adoption of new ideas, in which agents make no decisions.

In the mentioned framework, spatiotemporal dynamics are determined essentially by two parameters: the frequency of meetings, given by the rate of adoption $\alpha \in(0,1)$, and a parameter translating the fall in the probability to meet when distance between agents increases, $\delta>0$. This last parameter works in the following way: the probability that an agent located at point $\delta$ in space meets an agent at point $\ell$ ' is $\exp (-$ $\left.\delta\left|\ell-\ell^{\prime}\right|\right)$ times lower than the probability of meeting an agent located in the original point in space.

If agents are distributed uniformly throughout the space, we can split it into a given number of locations or points in space, say $N$, each one containing an identical share of agents. Let $G(\ell, \mathrm{t})$ be the share of agents at location $\ell$ and time $t$ that have not yet adopted the new idea. Recovering parameters $\alpha$ and $\delta$, and their respective meanings, the probability of not accessing the new idea in period $t+1$ conditional on not having accessed in period $t$, at location $\ell$, corresponds to

$$
G\left(\ell^{\prime}, t+1\right)=G\left(\ell^{\prime}, t\right)\left[\frac{\sum_{\ell=1}^{N} G(\ell, t) \exp \left(-\delta\left|\ell-\ell^{\prime}\right|\right.}{\sum_{\ell=1}^{N} \exp \left(-\delta\left|\ell-\ell^{\prime}\right|\right.}\right]^{\alpha}
$$

This difference equation allows characterizing a process of diffusion of ideas in space and time. Regardless of the initial state, knowledge will disseminate and, asymptotically, reach all points in space and every agent located at each point. The fundamental information the structure of analysis offers relates the speed of adjustment towards the steadystate of $100 \%$ adopters. As it should be obvious, the more frequent are the meetings among agents (larger $\alpha$ ) and the less localized the diffusion process is (smaller $\delta$ ), the faster will be the adjustment process.

The described simple structure of dissemination of ideas across time and space constitutes a solid benchmark from which one may start analyzing more sophisticated situations. For example, the mechanical process according to which a meeting gives place to the transmission of knowledge can be replaced by a complex interaction structure where the potential diffusion of knowledge might be dependent on some compatibility features among agents. The important point to stress is that the presented model is one viable way of taking together the impact of time and space when addressing the behavior of economic agents.

Obviously, there are many other ways of addressing Economics as a spatiotemporal science, and this area of knowledge may eventually succeed in adapting structures of analysis from other fields of study. One possibility is to make use of the techniques proposed by authors that study ecological systems. In Mistro et al. [3] and Rodrigues et al. [4], for instance, techniques aimed at describing environmental processes of diffusion are carefully developed. A successful adaptation of such modeling to Economics requires essentially the capacity to understand the specificity of economic relations relatively to other processes of interaction one observes in society and in nature.

\section{References}

1. Lucas RE (2009). Ideas and Growth. Economica 76: 1-19.

2. Comin DA, Dmitriev M, Rossi-Hansberg E (2012) The Spatial Diffusion of Technology. NBER working paper no. 18534

3. Mistro DC; Rodrigues LAD, Petrovskii S (2012) Spatiotemporal Complexity of Biological Invasion in a Space-and-Time-Discrete Predator-Prey System with the Strong Allee Effect. Ecological Complexity 9: 16-32.

4. Rodrigues LAD, Varriale MC, Godoy WAC, Mistro DC (2012) Spatiotemporal Dynamics of an Insect Population in Response to Chemical Substances. Ecological Complexity.

*Corresponding author: Orlando Gomes, Lisbon Higher Institute of Accounting and Administration (ISCAL/IPL), Av. Miguel Bombarda 20, 1069-035 Lisbon, Portugal, E-mail: omgomes@iscal.ipl.pt

Received December 19, 2012; Accepted December 22, 2012; Published December 26, 2012

Citation: Gomes O (2012) Spatiotemporal Modeling in Economics. J Appl Computat Math 2:e128. doi:10.4172/2168-9679.1000e128

Copyright: (c) 2012 Gomes O. This is an open-access article distributed under the terms of the Creative Commons Attribution License, which permits unrestricted use, distribution, and reproduction in any medium, provided the original author and source are credited. 